\title{
Propagation of Measurement Noise Through Backprojection Reconstruction in Electrical Impedance Tomography
}

\author{
Alejandro F. Frangi*, Associate Member, IEEE, Pere J. Riu, Senior Member, IEEE, Javier Rosell, and \\ Max A. Viergever, Member, IEEE
}

\begin{abstract}
A framework to analyze the propagation of measurement noise through backprojection reconstruction algorithms in electrical impedance tomography (EIT) is presented. Two measurement noise sources were considered: noise in the current drivers and in the voltage detectors. The influence of the acquisition system architecture (serial/semi-parallel) is also discussed. Three variants of backprojection reconstruction are studied: basic (unweighted), weighted and exponential backprojection. The results of error propagation theory have been compared with those obtained from simulated and experimental data. This comparison shows that the approach provides a good estimate of the reconstruction error variance. It is argued that the reconstruction error in EIT images obtained via backprojection can be approximately modeled as a spatially nonstationary Gaussian distribution. This methodology allows us to develop a spatial characterization of the reconstruction error in EIT images.
\end{abstract}

Index Terms-Backprojection reconstruction, electrical impedance tomography, error propagation theory, reconstruction error characterization.

\section{INTRODUCTION}

$\mathbf{E}$ LECTRICAL impedance tomography (EIT) is an imaging technique producing functional images of the body based on the electrical properties of tissues. Basically, EIT images depict the different organs of the body thanks to their different electrical impedances which provide the desired contrast. Data acquisition is relatively simple. A low-amplitude alternating current (ac) is successively injected across pairs of electrodes surrounding the body while the voltage differences measured between the remaining surface electrodes are recorded. The formation of the impedance image from boundary data can be stated as an inverse boundary value problem. Usually, only the real part of the impedance (the conductivity) is reconstructed. In this paper, we confine ourselves to conductivity images.

Manuscript received July 1, 2001; revised March 1, 2002. This work was supported in part by the Spanish DGICYT under Research Project PB93-0952. The work of A. F. Frangi was supported by a CIRIT Grant. Asterisk indicates corresponding author.

*A. F. Frangi is with the Departamento de Ingeniería Electrónica y Comunicaciones, Universidad de Zaragoza, María de Luna 1, E-50018 Zaragoza, Spain (e-mail: afrangi@posta.unizar.es). He is also with the Aragon Institute of Engineering Research (I3A), Universidad de Zaragoza, E-50018 Zaragoza, Spain.

P. J. Riu and J. Rosell are with the División de Instrumentación y Bioingeniería, Departamento de Ingeniería Electrónica, Universidad Politécnica de Cataluña, 08034 Barcelona, Spain.

M. A. Viergever is with the Image Sciences Institute, Utrecht University, 3508 GA Utrecht, The Netherlands.

Publisher Item Identifier 10.1109/TMI.2002.800612.
Since the inverse problem in EIT is nonlinear, only iterative reconstruction algorithms seem appropriate to provide accurate images. These algorithms are highly flexible, but thereby computationally expensive. A more serious disadvantage of iterative algorithms is that they suffer from convergence problems when applied to (noisy) acquired data since the inverse problem in EIT is very ill posed [1], [2]. A related class of reconstruction techniques includes the adaptive methods [3], [4], in which the applied current patterns are adjusted to get the best signal [5]-[8]. A second family of reconstruction algorithms is noniterative, based on a linearization of the equation that governs the potential distribution within the object. The most widely used algorithms of this class are based on a backprojection operation and do not reconstruct the absolute conductivity but the change between two different conductivity distributions usually computed as a conductivity ratio. A reference frame (a boundary condition set) is first measured. The change in measurements between this set and a second frame, the data frame, is backprojected to obtain an image of the conductivity change between the data and reference distributions. The linearization of the governing equations is valid to a good approximation when the conductivity change between the two backprojected frames is small. Even with this restriction, these algorithms have been the most popular [9] for producing in vitro and in vivo images [10]-[19]. In a recent survey [20] of clinical and experimental applications of EIT related to pulmonary applications, 37 out of 40 studies, from 12 different research groups, used backprojection algorithms. More recently, in the latest International Conference on Electrical Bio-Impedance [21], seven out of nine papers related to clinical or in vivo applications used backprojection algorithms. Notable exceptions were those studies based on adaptive EIT systems. Finally, although backprojection algorithms have been the most widespread, there exist also other noniterative algorithms with improved accuracy based on one-step Newton methods of which the NOSER algorithm [22] is probably the most well known.

This paper aims to characterize the reconstruction or estimation error in EIT images obtained via backprojection reconstruction. This estimation error can be regarded in subsequent postprocessing steps as image noise. In other imaging modalities, the properties of image noise have been investigated (see, for instance, comments on X-ray, ultrasonic, and nuclear medicine imaging in [23]). To our knowledge, the first published results about image noise in EIT are from Sinton et al. [24], who give experimental results on the reconstruction 
error distribution within EIT images. Although they are the first in reporting that the error is not uniform over the image domain they do not offer a theoretical analysis explaining this fact. Following these works, Wang et al. [25], [26] proposed two strategies for signal filtering at the data acquisition level in order to reduce the spatial nonuniformity of reconstruction error. Recently, Kaipio et al. [27], [28] have shown that reconstruction error is also not uniform when one uses more sophisticated statistical inversion algorithms in EIT. Although the approach by Kaipio et al. allows the computation of both variances and covariances of the reconstruction error, their results must be obtained by numerical simulation and not by closed-form expressions. Finally, it is remarkable that different approaches to image reconstruction lead to similar conclusions regarding the nonuniformity of the reconstruction error [28] although there exist differences depending on the particular priors assumed in regularizing the problem.

The purpose of this paper is to provide a theoretical framework for studying measurement noise propagation in EIT backprojection reconstruction. This framework allows us to model the spatial distribution of the reconstruction error in EIT images and provides an explanation to previous experimental observations [24].

The organization of the paper is as follows. In Section II, a model of measurement noise is introduced together with a brief overview of practical data collection systems. The analysis of reconstruction error or image noise will focus on the family of backprojection reconstruction algorithms. Section III discusses the three variants of backprojection reconstruction algorithms used in this paper. Section IV proposes an analytical treatment of measurement noise propagation in EIT backprojection reconstruction. The results from this theory are compared with those of simulated and experimental data in Sections V and VI. Finally, Section VII discusses the results and concludes the paper.

\section{NOISE SOURCES IN EIT ACQUISITION}

\section{A. Noise Sources}

The first stage in the formation of an EIT image is data acquisition. A typical strategy in EIT acquisition systems is to measure the boundary voltage that is produced by a current injected at the boundary of the imaged object. Fig. 1 shows a diagram indicating a four-wire measurement system and the sources of noise that we will consider in this paper. A constant-amplitude ac of root-mean-square (rms) value $I$ and frequency $f_{o}$ is applied through an electrode pair; the corresponding potential difference [rms value $(V)]$ is measured at a second electrode pair. This voltage is attributed to the transimpedance $Z$. Since the information about the resistive component is in the in-phase signal a coherent demodulator phase sensitive detector (PSD) is used to extract its amplitude [29]. The lowpass filter incorporated in the demodulator will limit the bandwidth according to the transfer function, $H_{D}(f)$. In the same figure, we include two equivalent error sources. They are attributed to electronic noise in current drivers $\left(\mathbf{i}_{\mathbf{n}}\right)$ and in voltage detectors $\left(\mathbf{v}_{\mathbf{n}}\right)$.

Noise in the current driver has a multiplicative effect in the measured voltage since this noise propagates through the imaged impedance distribution. Noise in the voltage detector

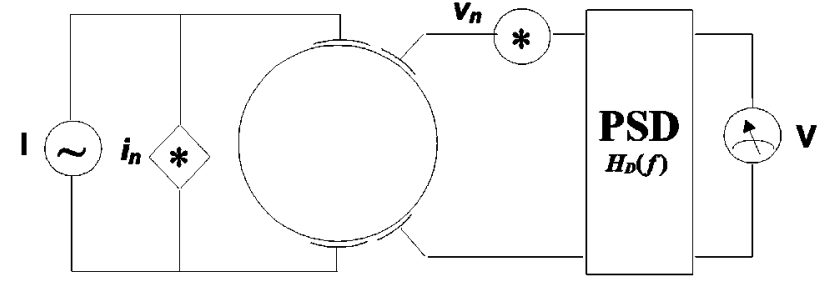

Fig. 1. Model of a four-wire measurement with the two noise sources analyzed in this paper. The phase-sensitive detector (PSD) gives a dc voltage that is proportional to the in-phase component of the measured impedance at the frequency of the injected ac current. A similar model would apply for the quadrature component.

electronics will have a signal-independent additive contribution mainly due to thermal noise. Meeson et al. [30] have suggested that, in addition to the mentioned sources, a third source of noise is due to the physiological variability of the measured transimpedance, $Z$. However, it is a nontrivial task to discriminate between "signal" and "noise" when both originate from physiological events. Such a distinction necessarily depends on the diagnostic purpose for which the images are collected. As the contribution of this transimpedance variability is application-dependent, it cannot be modeled in a generic way. For this reason, we have not considered this noise source in our analysis.

The system of Fig. 1 is described by the following equation, which is derived after coherent demodulation:

$$
V(t)=V+n(t)=|Z| \cos \varphi>I+v_{\mathbf{i}_{\mathbf{n}}}(t)+v_{\mathbf{v}_{\mathbf{n}}}(t)
$$

where $Z=|Z|_{\varphi}$ is the measured impedance (polar representation) at the frequency of the injected current, $n(t)$ is the noise in the measured voltage $V(t)$, and the subscript in the righthand-side voltages indicate the noise source. These voltages, although not explicitly indicated, make reference to in-phase lowpass equivalent signals. The measurement noise power under the assumption of uncorrelated noise sources is

$$
S_{\mathbf{n}}=S_{\mathbf{i}_{\mathbf{n}}}+S_{\mathbf{v}_{\mathbf{n}}}
$$

By assuming that both sources can be modeled as white Gaussian random processes with spectral densities $\eta_{\mathbf{i}_{\mathbf{n}}} / 2$ and $\eta_{v_{n}} / 2$, respectively, we obtain the following expressions for noise power:

$$
\begin{aligned}
& S_{v_{\mathbf{v}_{\mathbf{n}}}}=\int_{-\infty}^{\infty} \frac{\eta_{v_{\mathbf{n}}}}{2}\left|H_{D}(f)\right|^{2} d f=\eta_{v_{\mathbf{n}}} B_{T} \\
& S_{v_{\mathbf{v}_{\mathbf{n}}}}=\int_{-\infty}^{\infty} \frac{\eta_{\mathbf{k}_{\mathbf{n}}}}{2}\left|Z(f) H_{D}(f)\right|^{2} d f \approx \eta_{\mathbf{i}_{\mathbf{n}}}\left|Z\left(f_{o}\right)\right|^{2} B_{T}
\end{aligned}
$$

where $B_{T}$ is the noise equivalent bandwidth defined by [31] and $H_{D}(f)$ is the transfer function of the lowpass filter placed after the demodulator

$$
B_{T}=\left|H_{D}(f)\right|_{\max }^{-2} \int_{0}^{\infty}\left|H_{D}(f)\right|^{2} d f .
$$

The approximation introduced in (4) is reasonable if we consider that $B_{T}$, in EIT systems, is typically several hundred hertz 
while $f_{O}$ is typically above $10 \mathrm{kHz}$. Therefore, $B_{T} / f_{O} \ll 1$. This means that in the bandwidth of the system, the impedance that we are measuring will remain approximately constant. However, for example in industrial EIT applications this may not be the case.

The measurement signal-to-noise ratio $\left(\mathrm{SNR}_{m}\right)$ is defined by

$$
\begin{aligned}
\frac{1}{\mathrm{SNR}_{m}} & =\frac{S_{\mathbf{n}}}{S_{V}}=\frac{\left(\frac{\eta_{\mathrm{in}}}{2}\left|Z\left(f_{o}\right)\right|^{2}+\frac{\eta_{\mathrm{v}_{\mathbf{n}}}}{2}\right) B_{T}}{\left|Z\left(f_{o}\right)\right|^{2} \cos ^{2} \varphi I^{2}} \\
& =\frac{1}{\frac{\cos ^{2} \varphi I^{2}}{\frac{\eta_{\mathbf{i}_{\mathbf{n}}}}{2} B_{T}}}+\frac{1}{\frac{\left|Z\left(f_{o}\right)\right|^{2} \cos ^{2} \varphi I^{2}}{\frac{\eta_{\mathbf{V}} \mathbf{n}}{2} B_{T}}} \\
& =\frac{1}{\mathrm{SNR}_{i}}+\frac{1}{\mathrm{SNR}_{v}}
\end{aligned}
$$

where $\mathrm{SNR}_{i}$ and $\mathrm{SNR}_{v}$ denote the signal-to-noise ratios (SNRs) of the two sources of noise (current driver and voltage detector, respectively).

If noise in the current driver dominates, the $\mathrm{SNR}_{m}$ will be independent of the measured impedance (but not of the power of noise itself). For multiple electrode systems, like the ones used in EIT, this means that the noise will have a power that is proportional to the measured voltage (multiplicative noise) and, thus, will be different for each electrode. On the other hand, if noise is dominated by the voltage detector electronics, its power will be independent of the measured signal (additive noise) but the SNR will depend on the measured impedance and, in multiple electrode systems, will be different for different electrode pairs. The overall $\mathrm{SNR}_{m}$ will be, thus, limited by the measurement with the lowest signal level. We will refer to the situation that thermal or electrical noise in the voltage detectors dominates over that in the current driver by voltage detector dominated (VDD). The opposite case will be referred as current driver dominated (CDD) noise.

\section{B. EIT Data Collection Approaches}

There are two main approaches to EIT data collection, namely, serial [32]-[36] and semi-parallel [37]-[43] systems. This classification is based on the protocol used to collect voltage measurements [44] and they arise as a tradeoff between speed and complexity. Other less extended configurations have also been proposed: fully parallel [45] and adaptive [3], [4], [46]-[48] systems. The latter, however, requires special reconstruction algorithms that differ from the backprojection approach analyzed in this paper. For updated surveys on electrical impedance tomography systems, see [48] and [49].

In the sequel, we assume that the system is made up of $M$ equally spaced electrodes that sample the peripheral potential profile of the object to be imaged. Current is successively injected through all adjacent electrode pairs. With $M$ electrodes it is possible to obtain $(M-3)$ independent four-wire measurements per injection pair. We measure the differential voltage between adjacent electrode pairs. In this way, the voltage measurements are a rough approximation to the boundary potential gradient in the tangential direction.

1) Serial Acquisition Systems: Fig. 2 shows a block diagram of a serial acquisition system. Current is injected serially through adjacent electrode pairs (adjacent current

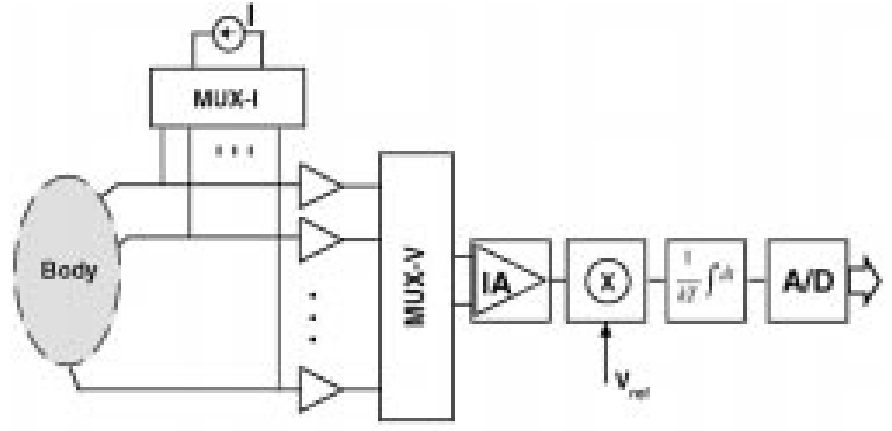

Fig. 2. A simplified block diagram of a serial acquisition system. A single acquisition channel is time-multiplexed for measuring the differential voltage between all pairs of adjacent electrodes. Integration intervals do not overlap between different detector electrode pairs even for those pairs belonging to the same injection pattern.

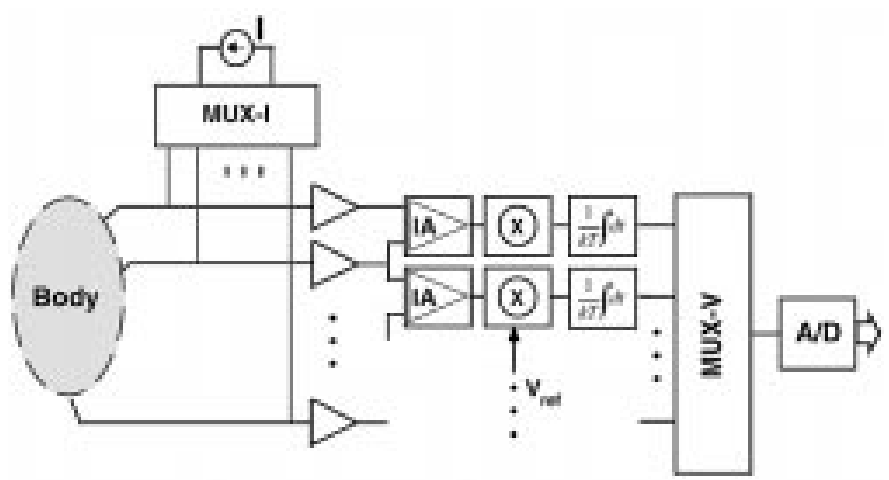

Fig. 3. A simplified block diagram of a semi-parallel acquisition system. Current injection is performed serially. For each injection pair, multiple acquisition channels record the voltages in parallel. Because of this simultaneous acquisition, the integration intervals are completely overlapped between channels. If the current driver is the dominant source of noise, noise in all parallel measurements will have the same origin and, thus, a strong correlation.

pattern). For each pattern, the differential voltages between adjacent electrodes are recorded serially by time-multiplexing a unique signal conditioning channel and analog-to-digital (A/D) converter. An important part of this channel is the coherent demodulator that incorporates an integrator block. The equivalent bandwidth $\left(B_{T}\right)$ of this block is the inverse of the acquisition time of each measurement. The integrator averages $k$ periods $\left(T=1 / f_{\circ}\right)$ of the measured differential voltage, thus increasing the SNR $\left(B_{T}=1 / k T\right)$. After a whole frame has been recorded, we obtain a matrix $\mathcal{V}=\left\{V^{j k} ; j, k=1 \ldots M\right\}$ of differential voltage measurements where $j$ and $k$ indicate, respectively, the injection and detection electrode pairs. Since the integration is carried out in nonoverlapping temporal intervals, the noise corrupting each one of the $M^{2}$ voltages is uncorrelated from that present in the remaining voltages of the frame.

2) Semi-Parallel Acquisition Systems: Fig. 3 shows a block diagram of a semi-parallel acquisition system. In this approach, current is driven in the same way as in the serial system. However, $M$ signal conditioning channels are incorporated. After coherent demodulation, we digitize the signal of each channel by time-multiplexing a unique $\mathrm{A} / \mathrm{D}$ converter. The main point here 
TABLE I

BACKPROJECTION ALGORITHMS ANALYZED IN THIS WORK

\begin{tabular}{ccccc}
\hline \hline Algorithm & $S\left(\sigma_{i} / \sigma_{r}\right)$ & $W^{j}(x, y)$ & $F\left(V_{i}^{j} / V_{r}^{j}\right)$ & $Q\left(V_{i}^{j} / V_{r}^{j}\right)$ Ref. \\
\hline Basic (BB) & $\ln \left(\sigma_{i} / \sigma_{r}\right)$ & 1 & $-Q\left(V_{i}^{j} / V_{r}^{j}\right)$ & $V_{i}^{j} / V_{r}^{j}-1[32]$ \\
Weighted (WB) & $\ln \left(\sigma_{i} / \sigma_{r}\right)$ & $-w^{j}(x, y)$ & $Q\left(V_{i}^{j} / V_{r}^{j}\right)$ & $\ln \left(V_{i}^{j} / V_{r}^{j}\right)[51]$ \\
Exponential (EB) & $\sigma_{i} / \sigma_{r}-1$ & 1 & $\exp \left\{-H^{j}(x, y) Q\left(V_{i}^{j} / V_{r}^{j}\right)\right\}-1 V_{i}^{j} / V_{r}^{j}-1$ & {$[53]$} \\
\hline \hline
\end{tabular}

The conductivity at a particular pixel $(x, y)$ is denoted by $\sigma$, where the subscripts $i$ and $r$ refer to the data and reference frames, respectively. The superscript $j$ denotes the projection number. $S$ is the conductivity change estimate. $Q$ is an approximation to the voltage change. $F$ is a function that is specific of each reconstruction algorithm and that maps the voltage change into a conductivity change. $W$ and $H$ are weighting functions that only depend on the pixel location $(x, y)$. For further details on the weighting functions $w$ and $h$, see the references.

is that, for each injection pair, integration is carried out simultaneously for all $M$ detection channels. In case of thermal and/or electrical noise and if voltage detector noise dominates over noise in the current driver, the noise contributions to all voltages in $\mathcal{V}$ will be essentially uncorrelated. However, the noise that is induced to the cords or measurement leads from external electric and magnetic fields is highly correlated between simultaneous measurements. Finally, if $\mathbf{i}_{\mathbf{n}}$ dominates over $\mathbf{v}_{\mathbf{n}}$, a large correlation between the measurements of each row in $\mathcal{V}$ will be observed.

The main advantage of semi-parallel systems over serial systems is their acquisition speed for a given SNR. On the other hand, serial systems have a lower cost since they only need a single signal conditioning channel.

\section{BACKPROJECTION FAMILY OF RECONSTRUCTION ALGORITHMS}

The backprojection operation assumes that the variation of the potential difference between two measuring electrodes (from data to reference frame) originates from a change in conductivity in the region bounded by the two equipotential lines ending in these electrodes [32]. If we assume that the current-driving electrodes are very close to each other, they can be approximated by a dipole. With this assumption the equipotential lines can be calculated for a uniform distribution [51]. It is assumed in these methods that the equipotential lines are not significantly distorted in the presence of a nonuniform distribution. In practice, multiple injection patterns are applied and their individual contributions (projections) are eventually averaged to form the conductivity change estimate of each pixel.

It is possible to differentiate two steps in the reconstruction process. For a given projection and pixel, we first select the boundary voltages that contribute to the formation of this particular pixel. For instance, we may use the nearest boundary measurement to the equipotential line running across that pixel. A more elaborated procedure is the one proposed by Santosa and Vogelius [52] that weights several measurements according to their distances to the equipotential line of the pixel being processed. This was the strategy implemented in this paper.

Once we have approximated the normalized tangential gradient of the voltage measurements at the objectťs boundary, it is necessary to somehow relate this quantity to the conductivity change. This second step is what differentiates the three approaches considered in this paper, namely, basic backprojection
(BB), weighted backprojection (WB), and exponential backprojection (EB).

A general formulation for the three reconstruction algorithms to be analyzed here is the following:

$$
S\left(\frac{\sigma_{i}}{\sigma_{r} ; x, y}\right)=\frac{1}{M} \sum_{j=1}^{M} W^{j}(x, y) F\left(\frac{V_{i}^{j}}{V_{r}^{j}}\right)
$$

where $S\left(\sigma_{i} / \sigma_{r} ; x, y\right)$ is the conductivity change estimate that depends on the contrast ratio, $\alpha=\sigma_{i} / \sigma_{r}$ at each pixel. $W^{j}(x, y)$ is a weighting function that only depends on the coordinates of the pixel being processed and the injection pattern. For a pixel at $(x, y), V_{i}^{j}$ and $V_{r}^{j}$ are the data and reference measurements from $\mathcal{V}_{i}$ and $\mathcal{V}_{r}$ that approximate the boundary voltage gradient. The superscript $j$ stands for the $j$ th projection. $F\left(V_{i}^{j} / V_{r}^{j}\right)$ is a function relating the ratio between the data and reference frames measurements of potential gradient with the conductivity. Table I characterizes the reconstruction algorithms that we consider in this paper.

After reconstruction, the resulting images are usually deblurred [32], [51], [52]. This can be viewed as a postprocessing step and has not been considered in this paper.

For the sake of simplicity, (7) assumes that for each pixel and projection only one voltage ratio $\left(V_{i}^{j} / V_{r}^{j}\right)$ was involved. This is a crude (nearest neighbor) approximation to the boundary voltage gradient. This leads to image artifacts whereby the equipotential areas related to each voltage detector become visible. To reduce this effect, we have applied the technique of Santosa and Vogelius [52] who proposed to average the voltage change, $Q$, of several electrode pairs. The function $Q$ depends on the type of backprojection algorithm. For BB and EB, the relative change is $\left(Q\left(V_{i}^{j} / V_{r}^{j}\right)=V_{i}^{j} / V_{r}^{j}-1\right)$; for $\mathrm{WB}$, the $\log$ change is applied $\left(Q\left(V_{i}^{j} / V_{r}^{j}\right)=\ln \left(V_{i}^{j} / V_{r}^{j}\right)\right)$. In Table I, we should replace the expression represented by $Q$ with the interpolated value

$$
Q\left(\frac{V_{i}^{j}}{V_{r}^{j}}\right)=\sum_{k} h_{k} Q\left(\frac{V_{i}^{j k}}{V_{r}^{j k}}\right)
$$

The weights, $h_{k}$, account for the distance between the location of the hypothetical equipotential line corresponding to the processed pixel (dashed line in Fig. 4) and the mid-point between the detection electrode pairs used to measure the boundary voltage gradient (solid lines). 


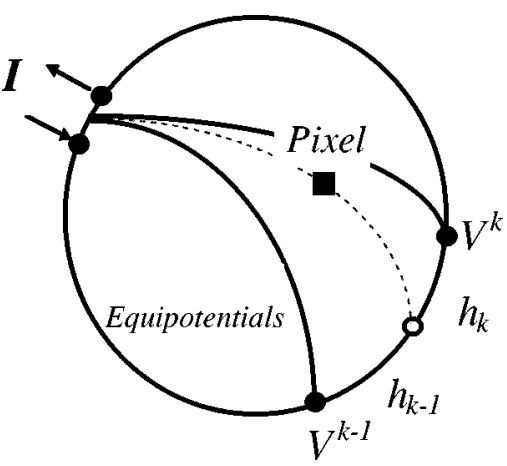

Fig. 4. In the backprojection implementation of santosa and vogelius, the parameter $Q$ is obtained through interpolation. The weights of the interpolation depend on the distance between the equipotential line of the processed pixel (dashed line) and the (discrete) differential measurements that sample the normalized tangential gradient at the object's surface (solid line).

\section{NOISE PROPAGATION}

\section{A. Statistical Distribution of Reconstruction Errors}

What type of errors corrupt EIT images when backprojection reconstruction is used? We argue that reconstruction errors are approximately normally distributed. Experimental evidence in this direction was previously reported in [54] based on observation of the histogram of pixels for a long record of reconstructed images. The following considerations give further support to this argument.

The set of boundary measurements is corrupted with thermal noise that is related to the electronics of the acquisition system. Thermal noise is known to be Gaussian distributed [55]. Each pixel is formed as a combination of the Gaussian distributed measurements [see (7)] corresponding to the different projections. This is true for WB and EB; for BB the combination is linear and the probability density function of the conductivity change estimate, $S$, will remain Gaussian. This is a well-known result of probability theory [55]. In WB and EB, the conductivity change estimate is the average of all projections; each one of them is a non-Gaussian random variable due to the nonlinear function appearing in its definition (see Table I). However, if the central limit theorem is applied to (7), we can expect that the final distribution of $S$ will tend to be normal as the number of projections increases.

In EIT acquisition systems, it is common to use a 16-electrode arrangement [50]. Although this value of $M$ might seem small to apply the central limit theorem, the normal distribution hypothesis appears to hold in both simulated and acquired images. This may be due to the fact that the SNR in EIT easily exceeds $40 \mathrm{~dB}$ (see, for instance, [23], [33], [34], and [56]). The nonlinearity introduced by the reconstruction algorithm $\left(F\left(V_{i}^{j} / V_{r}^{j}\right)\right)$ does not seem to alter the distribution substantially. If each projection has a distribution which only slightly deviates from a normal one, the projection sum will be similar to a normal distribution even with $M$ not very large.

Although the reconstruction errors may be considered approximately Gaussian for most practical purposes, one can argue that this will not be the case for pixels reconstructed with most terms $V_{i}^{j} / V_{r}^{j}$ being very large, e.g., when $V_{r}^{j}$ is small. In this case, the distributions of $S$ will be very skew unless $M$ is impractically large. In a recent paper, Kaipio et al. [28] have studied the marginal distribution of the reconstruction error using a statistical inversion technique. Although the reconstruction algorithm is essentially different to backprojection, they show that the marginal densities, although Gaussian-like, can be skew depending on the pixel's position within the image domain, its conductivity change, and the particular prior used to regularize the problem. The arguments given in previous paragraphs together with the observations of Kaipio et al. suggest that reconstruction errors are approximately Gaussian-distributed but that the accuracy of this approximation is affected by several factors. Therefore, this claim has to be interpreted with care.

Following a formal statistical treatment it is not feasible to derive a closed-form expression for the probability density function of $S$ in (7) to calculate its variance. In Section IV-B, we propose an approximated way of assessing the variance of the reconstruction error. This approach is essentially the same of Macovsky [23] for estimating noise variance of the line integral in X-ray imaging systems.

\section{B. Reconstruction Error Estimation Using Error Propagation Theory}

Error propagation theory comes from the field of measurement science. It is a first-order approximation for assessing the uncertainty of a given indirect observation as derived from an uncertain (noisy) direct measurement.

Let $\mathrm{x}$ be a direct (measured) observation with magnitude $X$. Let $\mathbf{y}$ be an indirect (computed) observation of magnitude $Y$ that is related to $\mathrm{x}$ by the function $\mathrm{y}=f(\mathrm{x})$. If $\mathrm{x}$ is a random variable with mean $\overline{\mathbf{x}}$ and variance $\operatorname{var}(\mathbf{x})$, the variance of $\mathbf{y}$ can be approximated by [57]

$$
\operatorname{var}(\mathbf{y}) \approx f^{\prime 2}(\overline{\mathbf{x}}) \operatorname{var}(\mathbf{x}), \quad \text { if } f^{\prime \prime}(\overline{\mathbf{x}}) \bar{m}_{3}(\mathbf{x}) f^{\prime}(\overline{\mathbf{x}}) \operatorname{var}(\mathbf{x})
$$

where $\bar{m}_{3}(\mathrm{x})$ is the third-order central moment of $\mathrm{x}$ and $f^{\prime}$ and $f^{\prime \prime}$ are the first- and second-order derivatives of $f(\mathrm{x})$. This expression comes from a Taylor expansion of $f(\mathrm{x})$ in a neighborhood of $\overline{\mathbf{x}}$. The condition imposes that the contribution of terms $O\left((\mathrm{x}-\overline{\mathrm{x}})^{3}\right)$ or higher can be disregarded [57]. This will be true if $\mathrm{x}$ is normally distributed since all moments will be zero except for the mean and the variance.

Consider now a more general case, with $2 M$ independent random variables $\mathbf{x}_{j}^{1}$ and $\mathbf{x}_{j}^{2}$ representing the direct measurements, and a single indirect observation $\mathbf{y}$. Suppose that $\mathbf{y}$ is computed as a weighted average of functions of the direct measurements

$$
\mathbf{y}=\sum_{j=1}^{M} \omega_{j} f_{j}\left(\mathbf{x}_{j}^{1}, \mathbf{x}_{j}^{2}\right) .
$$


In this case, which resembles the situation of the backprojection operation, we can generalize (9) [57]

$$
\begin{aligned}
\operatorname{var}(\mathbf{y}) \approx & \sum_{j} \omega_{j}^{2}\left(\partial_{\mathbf{x}_{j}^{1}} f_{j}\left(\overline{\mathbf{x}}_{j}^{1}, \overline{\mathbf{x}}_{j}^{2}\right)\right)^{2} \operatorname{var}\left(\mathbf{x}_{j}^{1}\right) \\
& +\sum_{j} \omega_{j}^{2}\left(\partial_{\mathbf{x}_{j}^{2}} f_{j}\left(\overline{\mathbf{x}}_{j}^{1}, \overline{\mathbf{x}}_{j}^{2}\right)\right)^{2} \operatorname{var}\left(\mathbf{x}_{j}^{2}\right) \\
& +\sum_{j} \omega_{j}^{2}\left(\partial_{\mathbf{x}_{j}^{1}} f_{j}\left(\overline{\mathbf{x}}_{j}^{1}, \overline{\mathbf{x}}_{j}^{2}\right)\right)\left(\partial_{\mathbf{x}_{j}^{2}} f_{j}\left(\overline{\mathbf{x}}_{j}^{1}, \overline{\mathbf{x}}_{j}^{2}\right)\right) \\
& \times \rho_{j}^{12} \sqrt{\operatorname{var}\left(\mathbf{x}_{j}^{1}\right)} \sqrt{\operatorname{var}\left(\mathbf{x}_{j}^{2}\right)}
\end{aligned}
$$

where $\rho_{j}^{12}$ is the cross-correlation between $\mathrm{x}_{j}^{1}$ and $\mathrm{x}_{j}^{2}$. In the literature on error propagation [57], the cross-terms in the previous expression are usually omitted which is equivalent to assuming that the random variables are uncorrelated $\left(\rho_{j}^{12}=0\right)$. If we now apply (11) to the three backprojection algorithms given by (7) and (8), we can derive the following general expressions for $\operatorname{var}(\mathbf{S})=N^{2}$. For BB, we obtain

$$
\begin{aligned}
N^{2}(x, y)= & \frac{1}{M^{2}} \sum_{j} \sum_{k, l} h_{k} h_{l} \rho_{k l l}\left(\frac{1}{\bar{V}_{r}^{j_{k}}}\right) \\
& \cdot\left(\frac{1}{\bar{V}_{r}^{j_{l}}}\right) \sqrt{\operatorname{var}\left(V_{i}^{j_{k}}\right)} \sqrt{\operatorname{var}\left(V_{i}^{j_{l}}\right)} \\
& +\frac{1}{M^{2}} \sum_{j} \sum_{k, l} h_{k} h_{l} \rho_{k l}\left(\frac{\bar{V}_{i}^{j_{k}}}{\bar{V}_{r}^{j_{k}}{ }^{2}}\right) \\
& \cdot\left(\frac{\bar{V}_{i}^{j_{l}}}{\bar{V}_{r}^{j_{l}}}\right) \sqrt{\operatorname{var}\left(V_{r}^{j_{k}}\right)} \sqrt{\operatorname{var}\left(V_{r}^{j_{l}}\right)}
\end{aligned}
$$

for WB

$$
\begin{aligned}
N^{2}(x, y)= & \frac{1}{M^{2}} \sum_{j} \sum_{k, l} h_{k} h_{l} \rho_{k l l}\left(\frac{W^{j}}{\bar{V}_{i}^{j_{k}}}\right) \\
& \cdot\left(\frac{W^{j}}{\bar{V}_{i}^{j_{l}}}\right) \sqrt{\operatorname{var}\left(V_{i}^{j_{k}}\right)} \sqrt{\operatorname{var}\left(V_{i}^{j_{l}}\right)} \\
& +\frac{1}{M^{2}} \sum_{j} \sum_{k, l} h_{k} h_{l} \rho_{k l}\left(\frac{W^{j}}{\bar{V}_{r}^{j_{k}}}\right) \\
& \cdot\left(\frac{W^{j}}{\bar{V}_{r}^{j_{l}}}\right) \sqrt{\operatorname{var}\left(V_{r}^{j_{k}}\right)} \sqrt{\operatorname{var}\left(V_{r}^{j_{l}}\right)}
\end{aligned}
$$

and finally, for EB

$$
\begin{aligned}
N^{2}(x, y)= & \frac{1}{M^{2}} \sum_{j} \exp \left[-2 H^{j} \sum_{m} h_{m}\left(\frac{\bar{V}_{i}^{j_{m}}}{\bar{V}_{r}^{j_{m}}}-1\right)\right] \\
& \times \sum_{k, l} h_{k} h_{l} \rho_{k l} \frac{H^{j^{2}}}{\bar{V}_{r}^{j_{k}} \bar{V}_{r}^{j_{l}}} \sqrt{\operatorname{var}\left(V_{i}^{j_{k}}\right)} \sqrt{\operatorname{var}\left(V_{i}^{j_{l}}\right)} \\
& +\frac{1}{M^{2}} \sum_{j} \exp \left[-2 H^{j} \sum_{m} h_{m}\left(\frac{\bar{V}_{i}^{j_{m}}}{\bar{V}_{r}^{j_{m}}}-1\right)\right] \\
& \times \sum_{k, l} h_{k} h_{l} \rho_{k l} \frac{H^{j^{2}} \bar{V}_{i}^{j_{k}} \bar{V}_{i}^{j_{l}}}{\bar{V}_{r}^{j_{k}} \bar{V}_{r}^{j_{l}^{2}}} \sqrt{\operatorname{var}\left(V_{r}^{j_{k}}\right)} \sqrt{\operatorname{var}\left(V_{r}^{j_{l}}\right) .}
\end{aligned}
$$

In the particular case of uncorrelated noise $\left(\rho_{l m}=\delta_{l m}\right)$ and without interpolation of the boundary voltage, the previous error estimates take simpler forms. For BB, we obtain

$$
\begin{aligned}
N^{2}(x, y)=\frac{1}{M^{2}} \sum_{j}\left(\frac{1}{\bar{V}_{r}^{j}}\right)^{2} \operatorname{var}\left(V_{i}^{j}\right) & \\
& +\frac{1}{M^{2}} \sum_{j}\left(\frac{\bar{V}_{i}^{j}}{\bar{V}_{r}^{j^{2}}}\right)^{2} \operatorname{var}\left(V_{r}^{j}\right)
\end{aligned}
$$

for WB

$$
\begin{aligned}
N^{2}(x, y)=\frac{1}{M^{2}} \sum_{j}\left(\frac{W^{j}}{\bar{V}_{i}^{j}}\right)^{2} \operatorname{var}\left(V_{i}^{j}\right) & \\
& +\frac{1}{M^{2}} \sum_{j}\left(\frac{W^{j}}{\bar{V}_{r}^{j}}\right)^{2} \operatorname{var}\left(V_{r}^{j}\right)
\end{aligned}
$$

and finally, for EB

$$
\begin{aligned}
N^{2}(x, y)= & \frac{1}{M^{2}} \sum_{j}\left(\frac{H^{j}}{\bar{V}_{r}^{j}}\right)^{2} \\
& \times \exp \left[-2 H^{j}\left(\frac{\bar{V}_{i}^{j}}{\bar{V}_{r}^{j}}-1\right)\right] \operatorname{var}\left(V_{i}^{j}\right) \\
& +\frac{1}{M^{2}} \sum_{j}\left(\frac{H^{j} \bar{V}_{i}^{j}}{\bar{V}_{r}^{j^{2}}}\right)^{2} \\
& \times \exp \left[-2 H^{j}\left(\frac{\bar{V}_{i}^{j}}{\bar{V}_{r}^{j}}-1\right)\right] \operatorname{var}\left(V_{r}^{j}\right) .
\end{aligned}
$$

In the subsequent sections, we will discuss the experimental set-up to evaluate the validity of these expressions under different acquisition strategies. For a serial system, the noise can always be assumed to be uncorrelated $\left(\rho_{l m}=\delta_{l m}\right)$, while for semi-parallel data collection it will be shown that this assumption will not always be valid. However, the estimation of the cross-correlation coefficient is nontrivial. In this situation, the expressions in (15)-(17), will be inaccurate.

\section{Simulations AND EXPERIMENTAl Measurement SetuP}

\section{A. Simulations}

1) Forward Problem: Simulated sets $\mathcal{V}_{i}$ and $\mathcal{V}_{r}$ were generated based on the series expansion for the potential derived by Pidcock et al. [58]. The geometry considered here is a two-dimensional circular background disc of radius $R$ and conductivity $\sigma_{b}$. Embedded in it, a circular perturbation of conductivity $\sigma_{p}$ and radius $r$ is placed at a distance $r_{c}$ from the center of the disc.

The sets $\mathcal{V}_{i}$ and $\mathcal{V}_{r}$ were generated for an equally spaced 16-electrode arrangement. We will assume that the reference frame concerns the simulation of a disc with conductivity equal to the background conductivity of the reference frame.

2) Noise Generation: Noise was incorporated in the simulated frames with a normally distributed random number generator. The noise sources introduced in Section II-A were simulated, that is, noise in the voltage detectors and in the current 


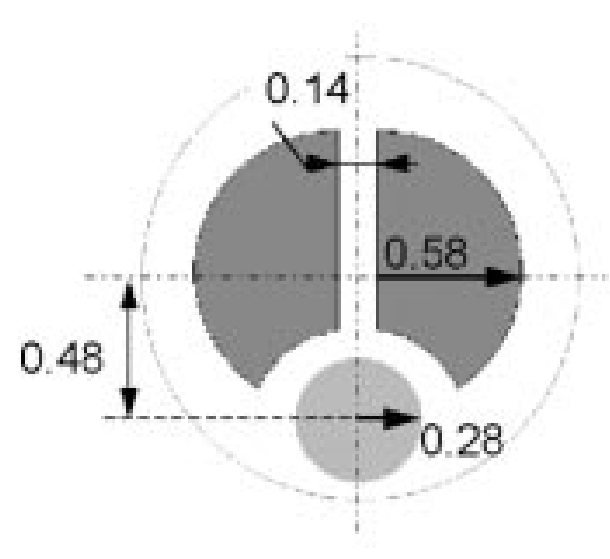

(a)

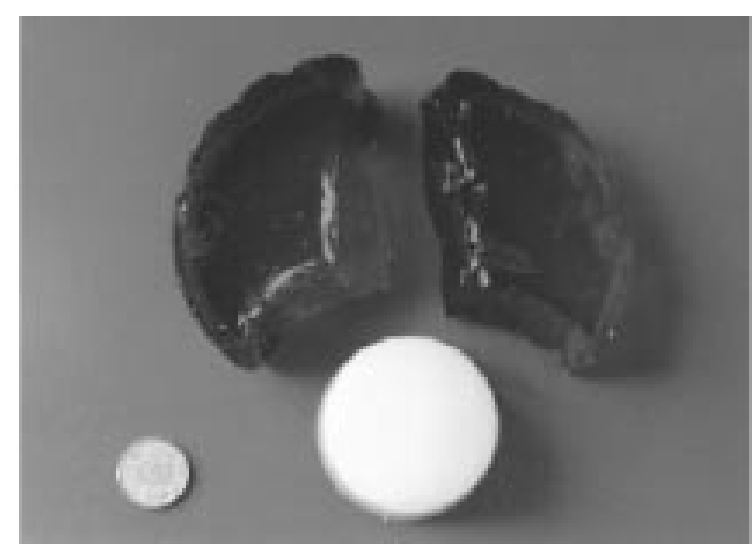

(b)

Fig. 5. Layout (a) and realization (b) of an agar-agar phantom of a thorax cross section. Two agar blocks simulating the lungs $(7 \mathrm{mS} / \mathrm{cm})$ and a smaller cylinder simulating the heart $(5 \mathrm{mS} / \mathrm{cm})$ were immersed in saline of conductivity $5.7 \mathrm{mS} / \mathrm{cm}$. The dimensions of the phantom are relative to the diameter of the PVC tank (ゆ19 $19 \mathrm{~cm})$.

driver. We have neglected possible noise arising from variations in the measured impedance due, for example, to physiological signals. Let $V^{j k}$ be an element of the measurement set $\mathcal{V}$. We used the following noise model [30], [59]:

$$
V^{j k}=z^{j k} I\left(1+\kappa_{n}^{j k}\right)+v_{n}^{j k}
$$

where $z_{j k}$ is the trans-impedance of the two-port defined with input in the $j$ th injection pair and output in the $k$ th detection pair. $I$ is the rms value of the injected current which is known. $\kappa_{n}^{j k}$ and $v_{n}^{j k}$ are random numbers which are, respectively, related to noise in the current driver and in the voltage detectors. In semi-parallel systems, $\kappa_{n}^{j k}=\kappa_{n}^{j}$. This means that noise present in all detection channels is correlated but it is uncorrelated from that present in the remaining projections of the frame.

For current driver noise, the $\mathrm{SNR}\left(\mathrm{SNR}_{i}\right)$ is

$$
\mathrm{SNR}_{\mathbf{i}}=\frac{I^{2}}{I^{2} \operatorname{var}\left(\kappa_{n}^{j k}\right)}=\frac{1}{\operatorname{var}\left(\kappa_{n}\right)} .
$$

The variance of $\kappa_{n}^{j k}$ was considered to be equal for all injection patterns since usually there is only one type of current source in a serial or semi-parallel acquisition system.

The definition of the SNR for the voltage detector $\left(\mathrm{SNR}_{v}\right)$ needs some explanation. The differential voltage around the object boundary decreases in amplitude as one moves away from the injection pair. For example, the ratio between the highest and lowest differential voltages of the profile corresponding to a uniform distribution of a 16-electrode system is around $18 \mathrm{~dB}$ (this was calculated based on Pidcock's expressions). Noise, however, does not depend on the voltage profile and has the same variance for all the measurements. These two facts make that the $\mathrm{SNR}_{v}$, will be different at each electrode [24]. We defined the $\mathrm{SNR}_{v}$ as the $\mathrm{SNR}$ in the electrode pair with the maximum voltage (four-wire measurement) in the reference frame

$$
\begin{aligned}
\mathrm{SNR}_{v}= & \frac{\max _{j, k}\left\{\left(V_{r}^{j k}\right)^{2} ; V_{r}^{j k} \in \mathcal{V}_{r}\right\}}{\operatorname{var}\left(\mathbf{v}_{n}^{j k}\right)} \\
= & \frac{\max _{j, k}\left\{\left(V_{r}^{j k}\right)^{2} ; V_{r}^{j k} \in \mathcal{V}_{r}\right\}}{\operatorname{var}\left(\mathbf{v}_{n}\right)}
\end{aligned}
$$

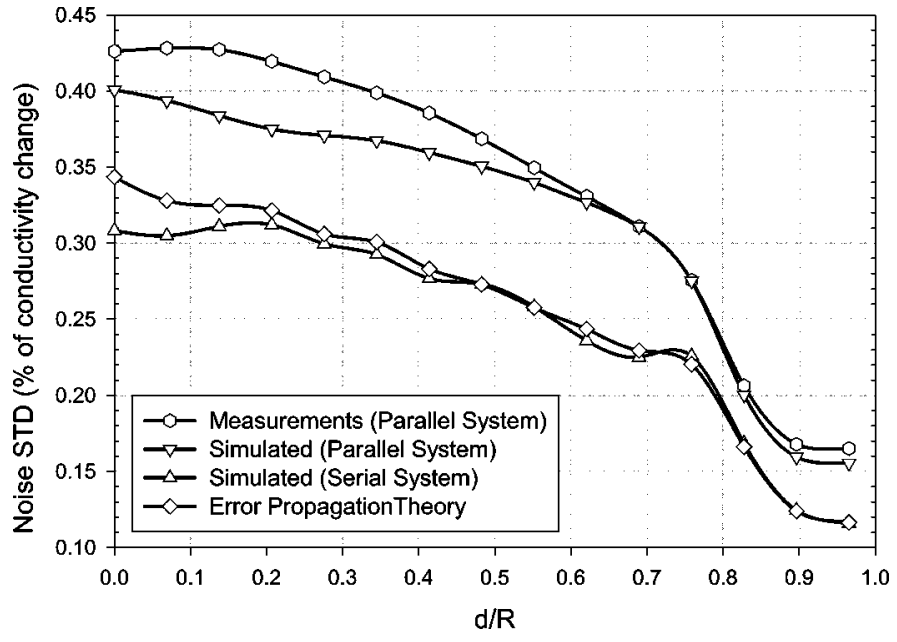

Fig. 6. Reconstruction error standard deviation profiles along the radial direction for EB. Error propagation theory underestimates SD noise in semi-parallel systems when it is CDD since the assumption of independence is not satisfied. The measurements were carried out with $T I E_{S y s}$ acquisition system with an integration time yielding $\mathrm{SNR}_{i}=48 \mathrm{~dB}$ and $\mathrm{SNR}_{v}=70 \mathrm{~dB}$.

where we considered that all acquisition channels have the same noise variance. If the acquisition system is properly designed, mismatches between the components in each acquisition channel are avoided and all of them will have a similar noise contribution.

\section{B. Measurements on a Uniform Distribution}

We have experimented with a real-time semi-parallel acquisition system called $T I E_{S y s}$ that was developed by our research group [56]. The injection frequency was fixed at $32 \mathrm{kHz}$. Currently this system has its dominant noise source in the current driver (CDD noise). The number of integration periods can be changed interactively in order to achieve a desired $\mathrm{SNR}_{m}$ at the cost of slowing the acquisition time.

The measurements were obtained from a uniform conductivity distribution consisting of $\mathrm{KCl}$ saline $\left(9 \mathrm{mS} / \mathrm{cm} @ 23{ }^{\circ} \mathrm{C}\right)$ in a PVC tank of 19-cm diameter and 4-cm height with the electrode plane at mid-height). A set of 2000 consecutive frames were acquired with $T I E_{S y s}$. The reciprocity error [60] was 

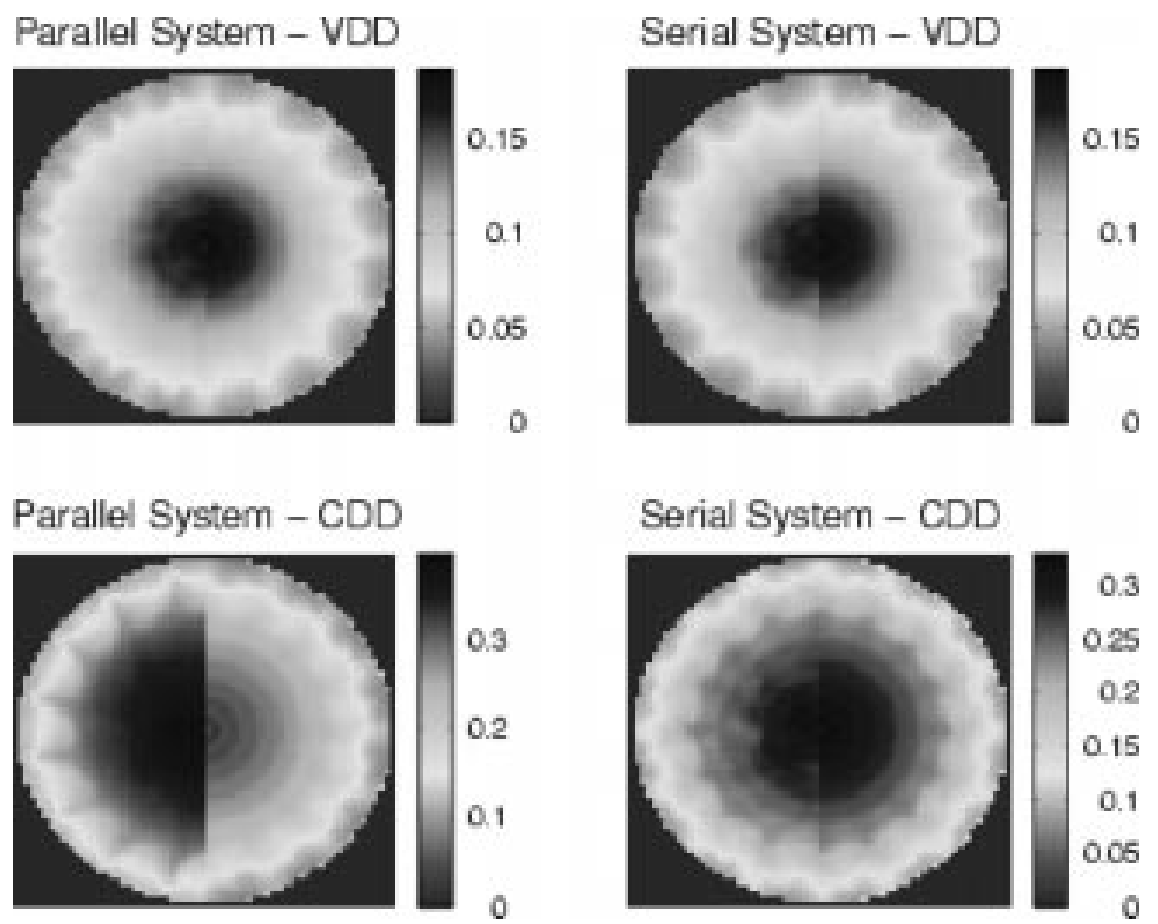

Fig. 7. Reconstruction error SD patterns for a uniform distribution $(\alpha=1)$ using EB. The left half portion of each pattern was obtained through semi-analytical simulation; the right half portion refers to the SD assessed through error propagation. Color bars indicate the SD of the reconstruction error in percentage of conductivity change. The example is based on the following SNRs: CDD noise, $\mathrm{SNR}_{i}=48 \mathrm{~dB}$ and $\mathrm{SNR}_{v}=70 \mathrm{~dB} ; \mathrm{VDD}$ noise, $\mathrm{SNR}_{i}=60 \mathrm{~dB}$ and SNR $v=$ $70 \mathrm{~dB}$.

below $3 \%$ in all measurements. The integration time was $k T=$ $2.18 \mathrm{~ms}$.

In these measurements, the SNR due to the current source $\left(\mathrm{SNR}_{i}\right)$ was $48 \mathrm{~dB}$ and that due to the voltage injectors $\left(\mathrm{SNR}_{v}\right)$ was $70 \mathrm{~dB}$. These values were estimated with the method described in the Appendix I.

\section{Measurements on an Agar-Agar Phantom of a Thorax Cross Section}

In order to analyze the effect of noise in more complex conductivity distributions, we designed a phantom mimicking a thorax cross section. The same PVC tank of Section V-B was filled with $\mathrm{KCl}$ saline of $5.7 \mathrm{mS} / \mathrm{cm}$. Five reference frames were measured with the tank filled up with the saline only. After that, three agar blocks simulating the lungs and the heart were immersed in the saline according to the layout shown in Fig. 5 (all distances are relative to the radius of the tank), and 100 data frames were measured. The water level was kept constant at $4 \mathrm{~cm}$ in all measurements.

Agar-agar phantoms are interesting in the sense that they allow simultaneous control of conductivity and shape of the objects. To fix a given conductivity we add a known quantity of $\mathrm{KCl}$ to agar powder, dissolve the mixture in distillate water, heat over $70^{\circ} \mathrm{C}$ and finally cool the agar until solidification. The final conductivity of the block was measured with an Hewlett-Packard HP4192A impedance analyzer in order to account for possible variations of the desired conductivity due to water evaporation while heating. The blocks corresponding to the lungs and heart had a conductivity of $7 \mathrm{mS} / \mathrm{cm}$ and $5 \mathrm{mS} / \mathrm{cm}$, respectively. The number of measured frames was small (100 frames) in order to have a small overall acquisi- tion time. This allows to disregard salt diffusion during the recording of the whole sequence.

In these measurements, the integration time was lower $(k T=$ $1 \mathrm{~ms}$ ) than that used in Section V-B. Accordingly, the SNR decreased. Using the procedure described in the Appendix I we estimated the noise in the current driver and in the voltage detectors to be $\mathrm{SNR}_{i}=41 \mathrm{~dB}$ and $\mathrm{SNR}_{v}=66 \mathrm{~dB}$, respectively. The reciprocity error was kept below $3 \%$ in all measurements.

\section{RESULTS}

Reconstruction error was first analyzed in images of uniform conductivity distribution $(\alpha=1)$ as in [24]. In this simple case, we compared the spatial distribution of the reconstruction error as predicted by error propagation theory with the results from numerical simulation and experimental data. This was done for all three backprojection algorithms (BB, WB, and EB).

Second, we studied the reconstruction error for a circular object in an otherwise uniform conductivity distribution. The same comparison (theory, simulation, and experiments) was carried out, again, for the three reconstruction algorithms.

Finally, the results obtained on a phantom of a thorax cross section were analyzed in the same fashion. We verified that certain conclusions, derived from simpler distributions, also apply for more complex images.

\section{A. Reconstruction Errors With a Uniform Distribution}

Fig. 6 shows image profiles of the standard deviation (SD) of the reconstruction error along radii intercepting an electrode (trans-electrode line). This profile corresponds to EB and similar plots are obtained for the other reconstruction strategies 

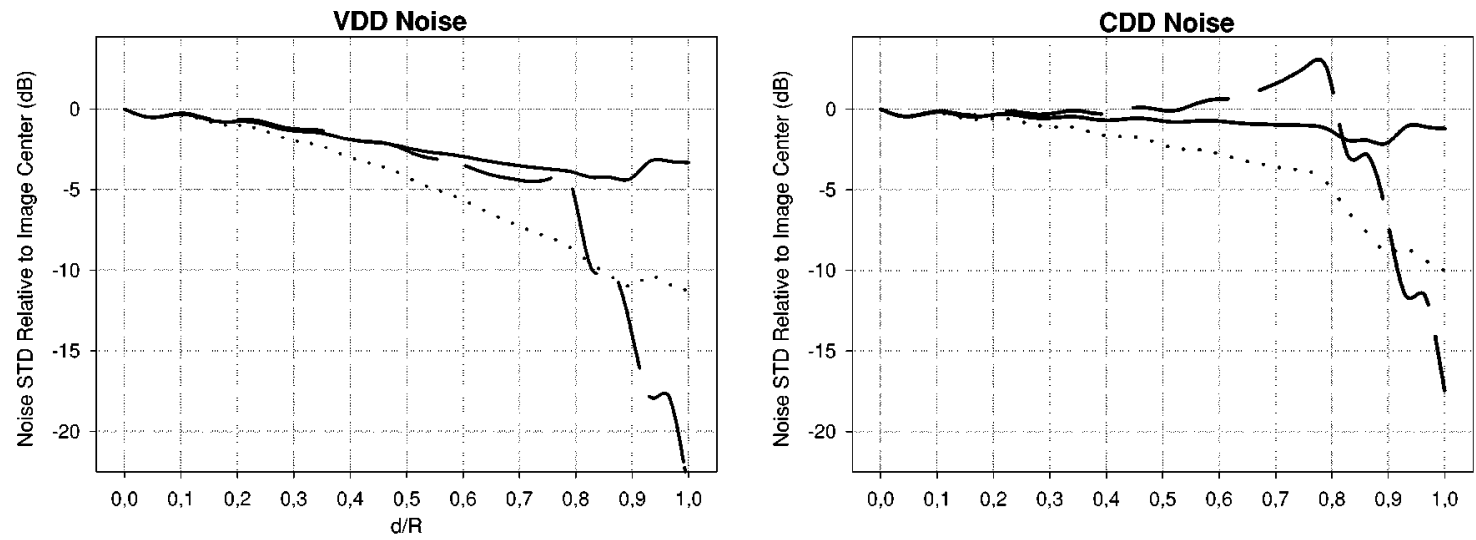

- Basic Backprojection $\quad---\quad$ Weighted Backprojection $\quad \cdots . . . . . . \quad$ Exponential Backprojection

Fig. 8. Comparison of SD of the reconstruction error for the three backprojection algorithms applying error propagation theory. Two measurements conditions were simulated: $\mathrm{CDD}$ noise $\left(\mathrm{SNR}_{i}=48 \mathrm{~dB}, \mathrm{SNR}_{v}=70 \mathrm{~dB}\right.$; bottom) and VDD noise ( $\mathrm{SNR}_{i}=60 \mathrm{~dB}, \mathrm{SNR}_{v}=70 \mathrm{~dB}$; top). The profiles correspond to a uniform conductivity distribution $(\alpha=1)$.

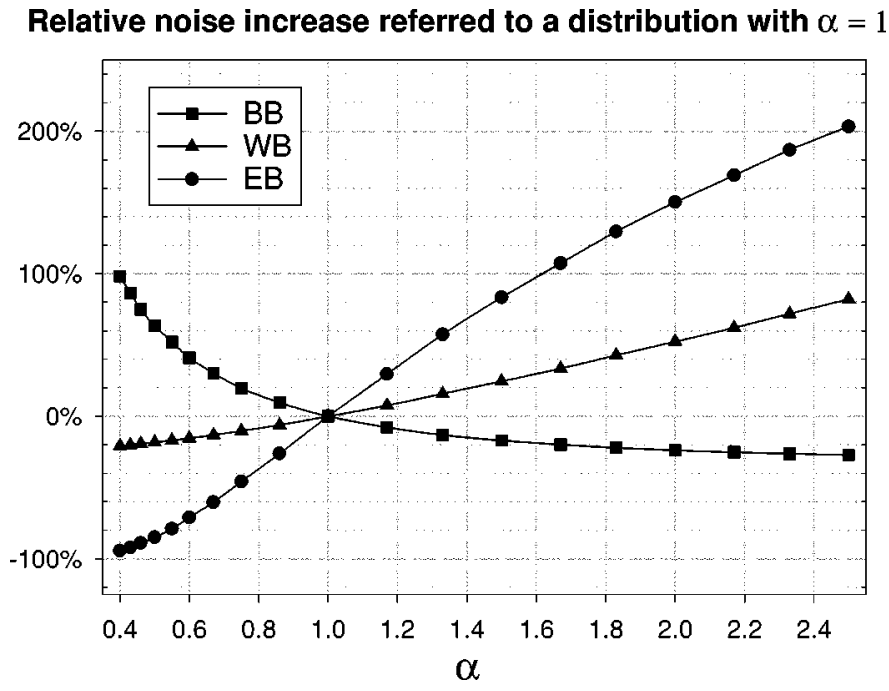

Fig. 9. Percent increase in reconstruction error (central pixel) as a function of contrast. Uniform distributions were considered. The simulation refers to a semi-parallel system with VDD noise $\left(\mathrm{SNR}_{i}=60 \mathrm{~dB}, \mathrm{SNR}_{v}=70 \mathrm{~dB}\right)$.

(BB and WB). The SNRs $\left(\mathrm{SNR}_{i}\right.$ and $\left.\mathrm{SNR}_{v}\right)$ used in the simulations were those estimated for our acquisition system (see Section V-B). This plot shows that error propagation underestimates the noise in semi-parallel systems with CDD noise. To further investigate this effect we simulated noisy measurements for the two possible data collection strategies (serial and semi-parallel systems), and noise conditions (CDD and VDD) and compared with the simulated error variance with that derived from error propagation. Fig. 7 shows the corresponding images of the standard deviation of the reconstruction error at each pixel. In order to visually compare the results from simulations with error propagation theory, the left half of the image displays the results from the former and the right half of the image those of the later.

Fig. 8 shows the variance of the reconstruction error relative to the center of the image for the three different reconstruction methods. For each algorithm, we simulated measurement noise under two measurement conditions: $\mathrm{CDD}\left(\mathrm{SNR}_{i}=48 \mathrm{~dB}\right.$; $\left.\mathrm{SNR}_{v}=70 \mathrm{~dB}\right)$ and $\mathrm{VDD}\left(\mathrm{SNR}_{i}=60 \mathrm{~dB} ; \mathrm{SNR}_{v}=70 \mathrm{~dB}\right)$ noise.
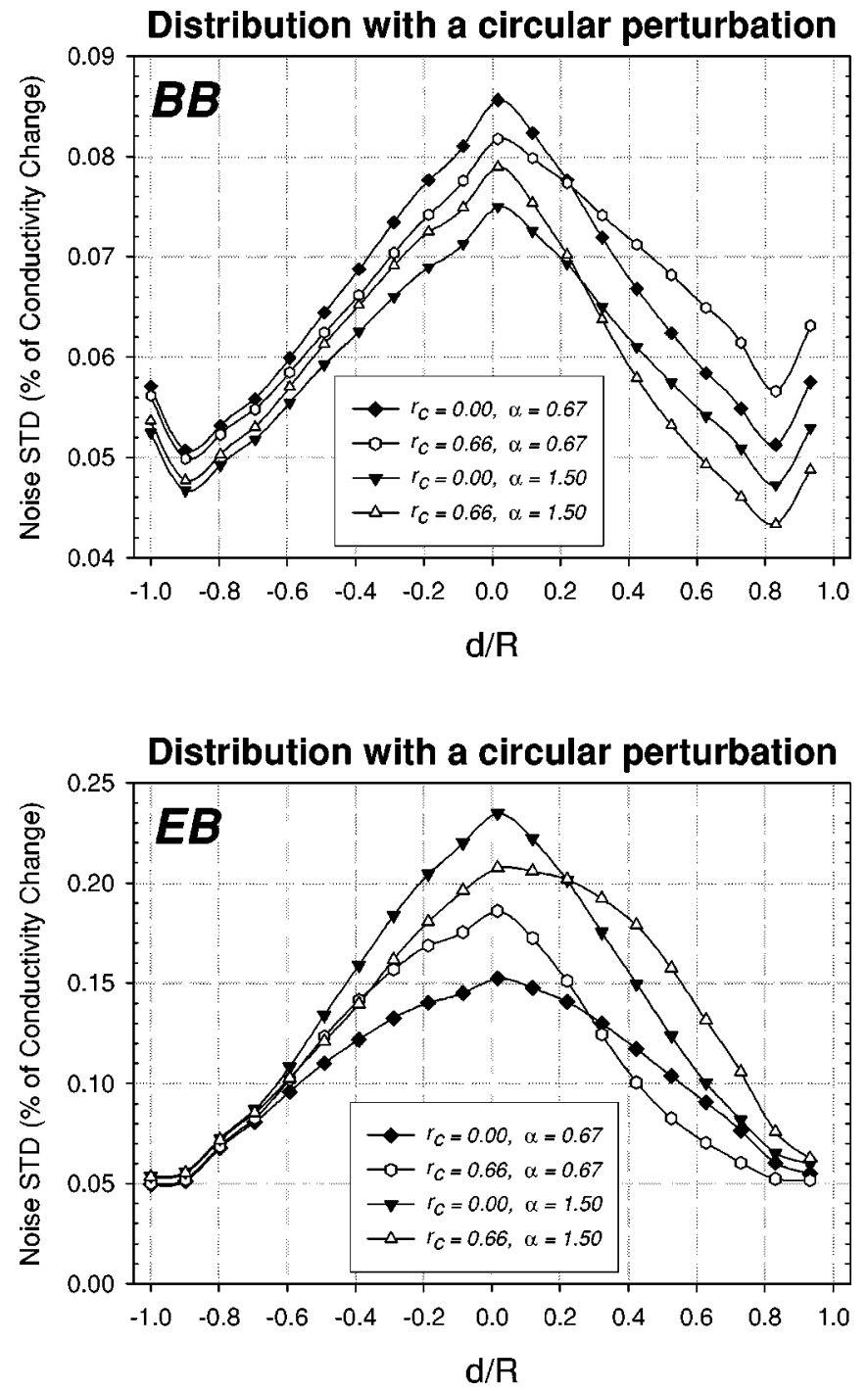

Fig. 10. Reconstruction error in a conductivity distribution with a circular perturbation. Simulated data was obtained considering a circular perturbation of $r / R=0.3$ and different eccentricities (here, $r_{c} / R=0.00$ and $r_{c} / R=0.66$ ) along a trans-electrode radial direction. These profiles refer to a semi-parallel system with VDD noise $\left(\mathrm{SNR}_{i}=60 \mathrm{~dB}, \mathrm{SNR}_{v}=70 \mathrm{~dB}\right)$. Compare with Fig. 9. 
BB Mean Image

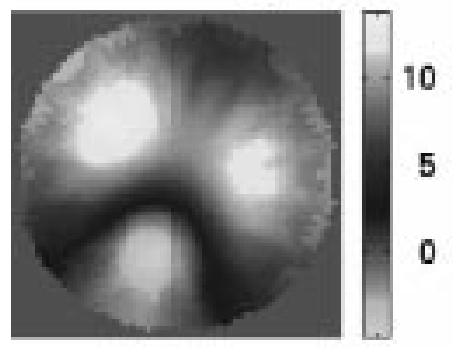

EB Mean Image

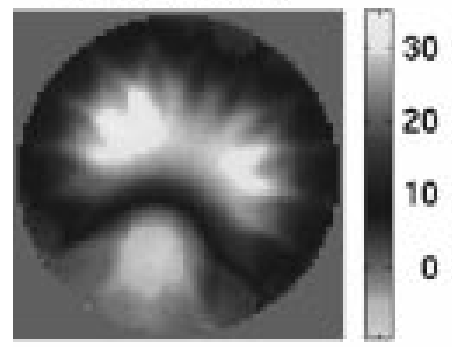

STD (Experimental)

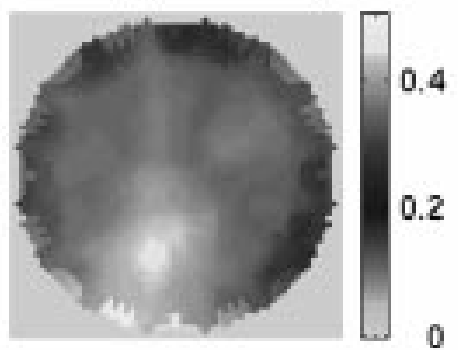

STD (Experimental)

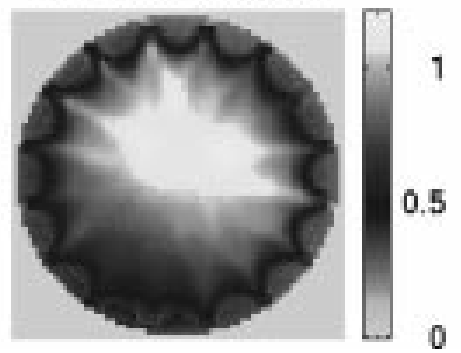

STD (Error Propagation)

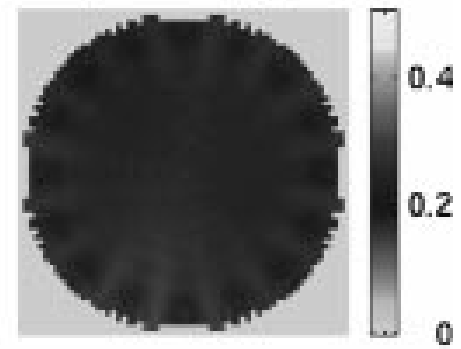

STD (Error Propagation)

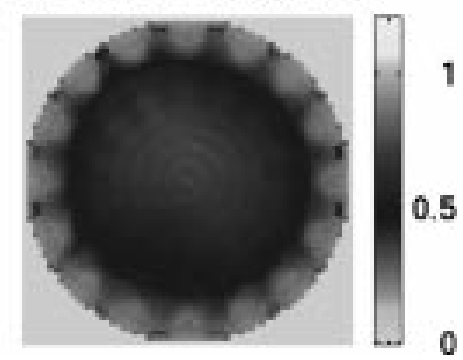

Fig. 11. Reconstruction error SD patterns in images from a phantom of a thorax cross section. A set of 100 frames was acquired with $T I E_{S y s}$, a semi-parallel acquisition system $\left(\mathrm{SNR}_{i}=41 \mathrm{~dB}\right.$ and $\left.\mathrm{SNR}_{v}=66 \mathrm{~dB}\right)$. In $\mathrm{BB}$, the error is higher in regions of conductivity decrease (heart) while in EB this happens in regions of conductivity increase (lungs).

Finally, Fig. 9 plots the relative change in reconstruction error $\mathrm{SD}$ at the center of the image as a function of the conductivity contrast between the reference and the data frames.

\section{B. Reconstruction Errors in the Presence of a Single Circular Perturbation}

Fig. 10 plots the profile of the reconstruction error SD along a diameter of the image $\left(\mathrm{SNR}_{i}=60 \mathrm{~dB}, \mathrm{SNR}_{v}=60 \mathrm{~dB}\right)$. The perturbation (with radius $r / R=0.3$ ) was displaced from the center toward the right along the radial direction. Two examples are shown for the object placed at the center and at $r_{c} / R=0.67$, and for two different contrasts (an increase and a decrease in conductivity).

\section{Reconstruction Errors in More Complex Conductivity Distributions}

The results of error propagation theory were also compared with the experimental data of the thorax cross-section phantom. This phantom had three objects simulating the two lungs and the heart. The lungs had a contrast of 1.23 and the heart of 0.88 (see Section V-C).

To apply error propagation formulae in this case we used the following procedure. The mean and standard deviation values of each of the voltages in the reference and data frames were obtained from the sequences described in Section V-C. With these values, we applied the expressions derived from error propagation theory. Fig. 11 shows the images obtained with BB and EB and the corresponding experimental and error propagation noise patterns.

\section{DISCUSSION AND CONCLUSION}

In Section IV-A, we gave some arguments supporting the hypothesis that the reconstruction error in EIT can be assumed to be Gaussian distributed. Moreover, in Section VI, it became apparent that simulations, experimental data, and error propagation theory convey to the conclusion that this error is spatially nonstationary and is dependent on the imaged conductivity distribution.

Figs. 6 and 7 showed that the reconstruction error estimate of error propagation theory, and that of simulated and experimental results are in good agreement. There are two exceptions to this. In the first place, Fig. 6 shows that the experimental noise profile is slightly above the corresponding simulated noise profile (for semi-parallel data collection). The average relative error between those profiles was $-7 \%$. This discrepancy could arise from other noise sources disregarded in the simulation, e.g., cross talk noise between different acquisition channels and interferences.

The largest disagreement between error propagation estimates and simulated/experimental results is for the particular case of a semi-parallel systems and CDD noise. In all the estimates derived from error propagation theory, we assumed a linear interpolation scheme of the boundary voltages. That is, the voltage measurement of a pixel is the linear interpolation of its two closest boundary voltages. Also, the correlation coefficient between the measured voltages was assumed to be zero (uncorrelated noise). This is a realistic assumption for a serial system and for a semi-parallel system with VDD noise. For a semi-parallel system with CDD noise, the correlation coefficient will be nonzero but it is difficult to estimate its exact value because it depends on the relative contribution of noise in the current drivers and voltage detectors. As a consequence, our derivations from error propagation theory under the assumption of uncorrelated noise will be inaccurate.

The spatial distribution of image noise is not homogeneous. For uniform distributions, the noise is mostly higher at the center 
of the image than toward the boundaries (Fig. 8) irrespective of the reconstruction algorithm and dominant noise source. The only exception is WB where the noise is higher at $75 \%$ of the radius for CDD noise. In the presence of a circular perturbation, the peak of the error is slightly shifted toward the perturbation but the error is essentially larger at the center than at the boundaries (Fig. 10).

The imaged conductivity distribution can modify the spatial distribution of the reconstruction error compared with an homogeneous distribution. Although it is difficult to draw conclusions for a generic conductivity distribution, it is possible to see some trends in the experiments. Fig. 9 shows that, even for an homogeneous distribution, there is an asymmetric response in the propagation of noise for two conductivity distributions of symmetric contrast (i.e., when the role of reference and data frames are exchanged). According to the results of Fig. 9 for WB and $\mathrm{EB}$, reconstruction error increases with contrast while $\mathrm{BB}$ has an opposite behavior. Reconstruction error SD in WB is the least sensitive to contrast for a conductivity decrease; $\mathrm{BB}$ is the least sensitive for a conductivity increase.

The thorax phantom provides a more realistic conductivity distribution where simultaneous increases and decreases of conductivity are present. Fig. 11 shows again that error propagation expressions underestimate the reconstruction error in semi-parallel systems with CDD noise. However, qualitative information about the spatial distribution of the error is still present in the corresponding error SD images. reconstruction error SD for EB reaches its maximum in the region of the lungs (a local increase in conductivity). For BB, in turn, the maximum is reached in the region corresponding to the heart (a local decrease in conductivity). Results for WB were not shown for parsimony but follow the same trends as those for EB. In summary, the spatial distribution of reconstruction errors in complex conductivity distributions is in agreement with the results for uniform distributions (Fig. 9).

EIT is a low-resolution technique and it is, thus, important not to introduce additional blurring in any postprocessing stage. Error propagation theory has been shown to produce a mathematical model of the spatial nonuniformity of reconstruction errors in EIT. This model could be use to introduce a priori information into an adaptive filter. In this way, the amount of blurring which generally accompanies filtering could be tuned to the amount of noise in each region.

The relationship between reconstruction error and regularization priors in EIT have been discussed recently by Kaipio et al. [27], [28]. Also, Tarantola [61] include a discussion on the covariance of the reconstruction error that is (partly) due to measurements errors. The above-mentioned references also indicate one of the basic issues in inverse problems: no inversion results can be obtained without a prior model. The problem in ad hoc inversion algorithms such as backprojection is that the prior model is implicit. For this reason, systematic exploration of estimation errors cannot be always carried out such as in Kaipio et al. [28]. The curious fact is that this implicit nature of backprojection is made explicit in the error propagation analysis carried out in this paper. However, a full characterization of the reconstruction errors would necessitate the computation or approximation of their covariances and not only their variances. Although co- variances are not considered in this paper, this information could explain also the spatial nature of the errors and the differences observed in Fig. 11. This would be clearly a worthwhile next step in fully understanding the structure of the estimation errors.

In summary, this paper presented a theoretical framework for the analysis of the propagation of measurement noise in EIT. This allowed us to predict the spatial distribution of reconstruction errors in EIT images. Furthermore, arguments were provided to support that the statistical distribution of EIT image errors are approximately Gaussian-distributed. This is not a consequence of the analytical treatment presented in this paper but agrees with previous experimental evidence. Finally, it was also mentioned that this approximation may not be valid under certain circumstances. We expect that a better understanding of the properties of reconstruction errors in EIT can help to introduce this knowledge into EIT image processing algorithms.

\section{APPENDIX}

In this section, we describe a simple method for assessing the SNR of a EIT system from a set of measurements. In particular, this method is useful in estimating the contribution of the two noise sources described in this paper.

A full measurement frame (reference or data frame) is made up of a matrix $\mathcal{V}$ of voltage measurements corresponding to a given conductivity distribution. Each row in $\mathcal{V}$ allocates the measurements from an injection pair and each column corresponds to each detection pair. In an ideal situation, this matrix should be diagonal as a consequence of the reciprocity theorem. If this is not the case, we will have a certain reciprocity error that can be evaluated as proposed in [60]. However, this kind of errors are mainly attributed to systematic (constant) errors that will not affect the estimate of the variance of the random noise but only the mean value of the measured voltage. If the reciprocity error is kept low in the measurements, its effect can be disregarded.

Another consideration is the number of useful measurements. For an $M$-electrode system only ( $M-3)$, voltages will be available in each row (four-wire measurements using adjacent injection).

Suppose now that we measure $N$ frames from the same conductivity distribution and that we obtain the mean $(\mathbf{V})$ and standard deviation $(\mathbf{S})$ matrices of the sequence $\left\{\mathcal{V}_{p} ; p=1 \ldots N\right\}$. The standard deviation matrix $\mathbf{S}$ accounts for the total measurement noise level (both CDD and VDD noise). CDD noise is dependent upon the signal level of the channel in which it is measured and VDD noise is independent of it. This can be formulated for the injector-detector pair $(j, k)$ in the following way:

$$
s_{T^{j k}}^{2}=s_{I}^{2} \cdot V_{j k}^{2}+s_{V}^{2}
$$

where $V_{j k}$ and $s_{T^{j k}}^{2}$ are elements of the matrices $\mathbf{V}$ and $\mathbf{S}$, respectively. On the other hand, $s_{I}^{2}$ and $s_{V}^{2}$ are, respectively, the standard deviation of CDD and VDD noise. $s_{T}^{2}$ is dimensionless because it is calculated assuming that the injected current is unity. By multiplying $s_{I}^{2}$ by the rms value of the injected current we obtain the noise in Amperes. It is a reasonable approximation to consider that $s_{I}^{2}$ and $s_{V}^{2}$ are independent of the injec- 
tion-detection pair. This is because in most EIT acquisition systems there is only one current-driver and each detector channel (for semi-parallel systems) is designed in such a way that mismatches are minimized.

Now, based on the matrices $\mathbf{V}$ and $\mathbf{S}$, and (21) we can set up a system of linear equations and solve for $s_{I}^{2}$ and $s_{V}^{2}$. This system is over-determined since we have $M(M-3)$ equations and only two unknowns. However, we can solve this system for the least-square estimate of the two unknowns.

That is, we form the vectors $\mathbf{v}_{\mathrm{sqr}}$ and $\mathbf{s}$ with the data (four-wire measurements only) in matrices $\mathbf{V}$ and $\mathbf{S}$ and the system matrix A

$$
\begin{aligned}
& \mathbf{A}=\left[\begin{array}{lll}
\mathbf{v}_{\mathrm{sqr}} & \vdots & \mathbf{1}
\end{array}\right]=\left[\begin{array}{cc}
V_{13}^{2} & \mathbf{1} \\
\vdots & \vdots \\
V_{j k}^{2} & \mathbf{1} \\
\vdots & \vdots \\
V_{M(M-1)}^{2} & \mathbf{1}
\end{array}\right] \\
& \mathbf{s}=\left[\begin{array}{c}
s_{T^{13}}^{2} \\
\vdots \\
s_{T^{j k}}^{2} \\
\vdots \\
s_{T_{M(M-1)}^{2}}^{2}
\end{array}\right]
\end{aligned}
$$

and solve the system for $s_{I}^{2}$ and $s_{V}^{2}$ in the least square sense

$$
\mathbf{s}=\mathbf{A}\left[\begin{array}{c}
s_{I}^{2} \\
s_{V}^{2}
\end{array}\right] \Longrightarrow\left[\begin{array}{c}
s_{I}^{2} \\
s_{V}^{2}
\end{array}\right]=\left(\mathbf{A}^{T} \mathbf{A}\right)^{-1} \mathbf{A}^{T} \mathbf{s}
$$

Finally, the SNRs are

$$
\begin{aligned}
\mathrm{SNR}_{i} & =\frac{1}{s_{I}^{2}} \\
\mathrm{SNR}_{v} & =\frac{\max _{k}\left\{v_{\mathrm{sqr}}^{k}\right\}}{s_{V}^{2}} .
\end{aligned}
$$

\section{ACKNOWLEDGMENT}

The authors would like to express their gratitude for the very useful comments of the reviewers to improve the clarity and presentation of this paper.

\section{REFERENCES}

[1] W. Breckon and M. Pidcock, "Ill-posedness and nonlinearity in electrical impedance tomography," in Information Processing in Medical Imaging, C. de Graaf and M. Viergever, Eds. New York: Plenun, 1988, pp. 235-44.

[2] M. Cheney and D. Isaacson, "Issues in electrical impedance imaging," IEEE Comput. Sci. Eng., vol. 2, no. 4, pp. 53-62, 1995.

[3] D. G. Gisser, D. Isaacson, and J. C. Newell, "Theory and performance of an adaptive current tomography system," Clin. Phys. Physiol. Meas., vol. 8, pp. 35-41, 1988.

[4] Q. Zhu, W. Lionheart, F. Lidgey, C. McLeod, K. Paulson, and M. Pidcock, "An adaptive current tomograph using voltage sources," IEEE Trans. Biomed. Eng., vol. 40, pp. 163-8, Feb 1993.

[5] K. Paulson, W. Lionheart, and M. Pidcock, "Optimal experiments in electrical impedance tomography," IEEE Trans. Med. Imag., vol. 12, pp. 681-6, Dec. 1993.
[6] A. Koksal and B. Eyuboglu, "Determination of optimum injected current patterns in electrical impedance tomography," Physiol. Meas., vol. 16, pp. A99-A109, 1995.

[7] M. Cheney, D. Isaacson, and J. Newell, "Electrical impedance tomography," SIAM Rev., vol. 41, pp. 85-101, 1999.

[8] W. Lionheart, J. Kaipio, and C. McLeod, "Generalized optimal current patterns and electrical safety in EIT," Physiol. Meas., vol. 22, pp. 85-90, 2001.

[9] D. C. Barber, "An overview on image reconstruction," in Clinical and physiological applications of Electrical Impedance Tomography, D. Holder, Ed. London, U.K.: Univ. College London Press, 1993, ch. 4.

[10] D. Holder and B. Brown, "Biomedical applications of EIT: a critical review," in Clinical and physiological applications of electrical impedance tomography, D. Holder, Ed. London, U.K.: Univ. College London Press, 1993, ch. 2.

[11] H. Jongschaap, R. Wytch, J. Hutchison, and V. Kulkarni, "Electrical impedance tomography: a review of current literature," Eur. J. Radiol., vol. 18, pp. 165-74, 1994.

[12] G. Hahn, I. Sipiková, F. Baisch, and G. Hellige, "Changes in the thoracic impedance distribution under different ventilatory conditions," Physiol. Meas., vol. 16, pp. 161-73, 1995 .

[13] B. Eyüboglu, A. Oner, U. Baysal, C. Biber, A. Keyf, U. Yilmaz, and Y Erdogan, "Application of electrical impedance tomography in diagnosis of emphysema-a clinical study," Physiol. Meas., vol. 16, pp. 191-211, 1995.

[14] R. Erol, R. Smallwood, B. Brown, P. Cherian, and K. Bardhan, "Detecting oesophageal-related changes using electrical impedance tomography," Physiol. Meas., vol. 16, pp. 143-52, 1995.

[15] S. Nour, Y. Mangnall, J. Dickson, J. A. G, and R. Pearse, “Applied potential tomography in the measurement of gastric emptying in infants," J Pediatr. Gastroenterol. Nutr., vol. 20, no. 1, pp. 65-72, 1995.

[16] I. Jürgens, J. Rosell, and P. Riu, "Electrical impedance tomography of the eye: in vitro measurements of the cornea and the lens," Physiol. Meas., vol. 17, pp. 187-95, 1996.

[17] D. Holder, A. Rao, and Y. Hanquan, "Imaging of physiologically evoked responses by electrical impedance tomography with cortical electrodes in the anaesthetized rabbit," Physiol. Meas., vol. 17, pp. 179-86, 1996.

[18] A. Vonk Noordegraaf, P. Kunst, A. Janse, R. Smulders, R. Heethaar, P. Postmus, T. Faes, and P. de Vries, "Validity and reproducibility of electrical impedance tomography for measurement of calf blood flow in healthy subjects," Med. Biol. Eng. \& Comp., vol. 35, no. 2, pp. 107-12, 1997.

[19] A. Vonk Noordegraaf, T. Faes, A. Janse, J. Marcus, J. Bronzwaer, P. Postmus, and P. de Vries, "Noninvasive assessment of right ventricular diastolic function by electrical impedance tomography," Chest, vol. 115 , no. 5, pp. 1222-8, 1997.

[20] I. Frerichs, "Electrical impedance tomography (eit) in applications related to lung and ventilation: a review of experimental and clinical activities," Physiol. Meas., vol. 21, pp. R1-21, 2000.

[21] Proc. XI Int. Conf. Electrical Bio-impedance, 2001.

[22] M. Cheney, D. Isaacson, J. Newell, J. Goble, and S. Simske, "NOSER: An algorithm for solving the inverse conductivity problem," Int. J. Imag Syst. Technol., vol. 2, pp. 66-75, 1990.

[23] A. Macovsky, Medical imaging systems. NJ: Prentice-Hall, 1983.

[24] A. M. Sinton, B. H. Brown, D. C. Barber, F. J. McArdle, and A. D. Leathard, "Noise and spatial resolution of a real-time electrical impedance tomograph," Clin. Phys. Physiol. Meas., vol. 12, pp. 125-30, 1992.

[25] W. Wang, B. H. Brown, A. D. Leathard, and L. Lu, "Noise equalization within EIT images," Physiol. Meas., vol. 15, pp. 211-6, 1994.

[26] W. Wang, B. H. Brown, and D. C. Barber, "Signal processing for noise equalization within EIT images," Inst. Elect. Eng. Coll. Innovations in Instrumentation for Electrical Tomography Dig. , vol. 1995/099, 1995.

[27] J. Kaipio, V. Kolehmainen, E. Somersalo, and M. Vaukonene, "Inverse problems with structural prior information," Inverse Prob., vol. 15, pp. 713-29, 1999.

[28] J. Kaipio, V. Kolehmainen, M. Vaukonene, and E. Somersalo, "Statistical inversion and Monte Carlo sampling methods in electrical impedance tomography," Inverse Prob., vol. 16, pp. 1487-1522, 2000.

[29] R. Smith, I. Freeston, B. Brown, and A. Sinton, "Design of a phase-sensitive detector to maximize signal-to-noise ratio in the presence of gaussian wideband noise," Meas. Sci. Technol., vol. 3, pp. 1054-1062, 1992.

[30] S. Meeson, B. Blott, and A. Killingback, "EIT data noise evaluation in the clinical environment," Physiol. Meas., vol. 17, pp. A33-8, 1996.

[31] A. B. Carlson, Communication systems: An introduction to signals and noise in electrical communication, 3rd ed. New York: McGraw-Hill, 1986. 
[32] D. C. Barber and B. H. Brown, "Imaging spatial distributions of resistivity using applied potential tomography-APT," in Information Processing in Medical Imaging, F. Deconinck, Ed. Zoetermeer, The Netherlands: Martinus Nijhoff, 1984, pp. 446-62.

[33] B. H. Brown and A. D. Seagar, "The Sheffield data collection system," Physiol. Meas., vol. 8, pp. 91-7, 1987.

[34] P. Riu, "Detecciò d'estructures estátiques en el cos humá usant mètodes multifreqüència en tomografía d'impedància elèctrica," Ph.D. dissertation, Polytechnic Univ. Catalonia, Barcelona, Spain, 1991.

[35] G. Cusick, D. Holder, A. Birquett, and K. Boone, "A system for impedance imaging epilepsy in ambulatory human subjects," Innov. Tech. Biol. Med., vol. 15, no. 1, pp. 33-9, 1994.

[36] E. Gersing and M. Osypka, "EIT using magnitude and phase in an extended frequency range," Physiol. Meas., vol. 15, pp. 21-8, 1994.

[37] R. W. M. Smith, "Design of a real-time impedance imaging system for medical applications," Ph.D. dissertation, University of Sheffield, Sheffield, UK, 1990

[38] M. Osypka and E. Gersing, "Parallel signal processing and multi-electrode current feeding in a multi-frequency EIT system," Innov. Tech. Biol. Med., vol. 15, no. 1, pp. 56-61, 1994.

[39] B. Blad, "An electrical impedance tomography system for complex impedance imaging," Innov. Tech. Biol. Med., vol. 15, no. 1, pp. 80-8, 1994.

[40] R. Smith, I. Freeston, and B. Brown, "A real-time electrical impedance tomography system for clinical use - design and preliminary results," IEEE Trans. Biomed. Eng., vol. 42, pp. 133-40, Feb. 1995

[41] B. Ayeva, "Etude et mise en oeuvre d'un appareillage de spectro-tomographie d'impédance," Ph.D., Diplome Universitaire de Recherche de l'Université Paul Sabatier, Tolouse, France, 1995.

[42] O. Casas, J. Rosell, R. Bragós, A. Lozano, and P. Riu, "A parallel broad-band real-time system for electrical impedance tomography," Physiol. Meas., vol. 17, pp. 1-6, 1996.

[43] P. Riu, R. Bragós, and J. Rosell, "Broadband quasidifferential multifrequency electrical impedance imaging system," Physiol. Meas., vol. 17, pp. 39-47, 1996.

[44] J. Rosell, D. Murphy, R. Pallás-Areny, and P. Rolfe, "Analysis and assessment of errors in a parallel data acquisition system for electrical impedance tomography," Physiol. Meas., vol. 9, pp. 93-9, 1988

[45] D. Murphy and P. Rolfe, "Aspects of instrumentation design for impedance imaging," Clin. Phys. Physiol. Meas., vol. 8, pp. 5-14, 1988.

[46] Q. Zhu, C. McLeod, C. Denyer, F. Lidgey, and W. Lionheart, "Development of a real-time adaptive current tomograph," Physiol. Meas., vol. 15 , pp. 37-43, 1994.
[47] R. Cook, G. Saulnier, D. Gisser, J. Goble, J. Newell, and D. Isaacson, "ACT3: a high-speed, high-precision electrical impedance tomograph," IEEE Trans. Biomed. Eng., vol. 41, pp. 713-22, Aug. 1994.

[48] P. Edic, G. Saulnier, J. Newell, and D. Isaacson, "A real-time electrical impedance tomograph,” IEEE Trans. Biomed. Eng., vol. 42, pp. 849-59, Sept. 1995.

[49] B. Rigaud, J.-P. Morucci, D. C. Barber, and B. H. Brown, "Report of a survey on electrical impedance tomography systems," INSERM Unitè 305, Tolouse, France, 1994.

[50] B. Rigaud and J.-P. Morucci, "Bioelectrical impedance techniques in medicine. Part III: Impedance imaging," Crit. Rev. Biomed. Eng., vol. 24, no. 4-6, pp. 467-8, 1996.

[51] D. C. Barber and B. H. Brown, "Recent developments in Applied Potential Tomography-APT," in Information Processing in Medical Imaging, S. L. Bacharach, Ed. Zoetermeer, The Netherlands: Martinus Nijhoff, 1986, pp. 106-12.

[52] F. Santosa and M. Vogelius, "A backprojection algorithm for electrical impedance imaging," SIAM J. Appl. Math., vol. 50, pp. 216-43, 1990.

[53] R. Povill and P. Riu, "Quantification in multifrequency tomography," Physiol. Meas., vol. 16, pp. 69-78, 1995.

[54] A. M. Sinton, B. H. Brown, D. C. Barber, F. J. McArdle, and R. W. M. Smith, "Noise and spatial resolution in images from a real-time electrical impedance tomograph," in Proc. IEEE EMBS 13th Annu. Conf., vol. 13, Orlando, FL, 1991, pp. 10-1.

[55] A. Papoulis, Probability random variables and stochastic processes. Tokyo, Japan: McGraw-Hill, 1965.

[56] J. Rosell, "Tomografía de impedancia eléctrica para aplicaciones médicas," Ph.D., Polytechnic University of Catalonia, Barcelona, Spain, 1989.

[57] N. C. Barford, Experimental measurements: precision, error and truth, 2nd ed. New York: Wiley, 1985.

[58] M. K. Pidcock, M. Kuzuoglu, and K. Leblebicioglu, "Analytical and semianalytical solutions in electrical impedance tomography. I: Two dimensional problems," Physiol. Meas., vol. 16, pp. 77-90, 1995.

[59] A. Adler and R. Guardo, "Electrical impedance tomography: regularized imaging and contrast detection," IEEE Trans. Med. Imag., vol. 15, pp. 170-179, Apr. 1997.

[60] J. Rosell, P. Riu, A. Lozano, and R. Pallás-Areny, "Errors and sensitivity in multielectrode systems for electrical impedance tomography," in Proc. 5th Mediterranean Conf. Medicine and Biological Engineering, MEDICON'89, Patras, Greece, Aug.-Sept. 29-1, 1989.

[61] A. Tarantola, Inverse problem theory. Amsterdam, The Netherlands: Elsevier, 1987. 